\title{
Le Nouveau visage de l'euroscepticisme conservateur à la Chambre des Communes
}

The New Face of Conservative Euroscepticism in the House of Commons

\section{Agnès Alexandre-Collier}

\section{OpenEdition}

\section{Journals}

Édition électronique

URL : http://journals.openedition.org/rfcb/1347

DOI : $10.4000 /$ rfcb. 1347

ISSN : 2429-4373

Éditeur

CRECIB - Centre de recherche et d'études en civilisation britannique

\section{Référence électronique}

Agnès Alexandre-Collier, « Le Nouveau visage de l'euroscepticisme conservateur à la Chambre des Communes », Revue Française de Civilisation Britannique [En ligne], XXII-2 | 2017, mis en ligne le 30 mai 2017, consulté le 01 mai 2019. URL : http://journals.openedition.org/rfcb/1347 ; DOI : 10.4000/ rfcb. 1347

Ce document a été généré automatiquement le 1 mai 2019.

Revue française de civilisation britannique est mis à disposition selon les termes de la licence Creative Commons Attribution - Pas d'Utilisation Commerciale - Pas de Modification 4.0 International. 


\section{Le Nouveau visage de l'euroscepticisme conservateur à la Chambre des Communes}

The New Face of Conservative Euroscepticism in the House of Commons

Agnès Alexandre-Collier

\section{Introduction}

1 Le résultat du référendum du 23 juin 2016 n'aura jamais été autant débattu par les commentateurs de toutes sortes, journalistes et universitaires. Le parti conservateur, au pouvoir depuis 2010 et dirigé par celui qui a été l'instigateur de ce scrutin, David Cameron, s'est tout particulièrement retrouvé dans la ligne de mire. C'est comme si les clivages autour de l'enjeu européen, dont les spécialistes expliquent pourtant qu'ils ne cessent de s'accentuer depuis une vingtaine années, avaient fait imploser le parti de manière totalement inopinée. Ceux qui ont toujours étudié le parti conservateur savent pourtant que ce résultat faisait partie des effets escomptés, sans pour autant oser vraiment l'imaginer, compte tenu de l'ampleur des répercussions possibles. David Cameron lui-même n'aurait jamais pu prévoir les conséquences du scrutin sur son propre sort, péchant sans doute par excès de confiance et d'optimisme.

2 Au retour des négociations avec ses partenaires européens et à l'approche du scrutin, le leader conservateur espérait pouvoir compter à la fois sur le soutien de ses proches et sur la loyauté légendaire de son groupe parlementaire, loyauté dont Lord Kilmuir (David Maxwell-Fyfe) avait dit, dans les années 1960, qu'elle était "l'arme secrète" des Conservateurs ${ }^{1}$. La victoire du parti en mai 2015 pouvait lui faire espérer, malgré l'euroscepticisme croissant de son parti, un ralliement de ses troupes derrière sa personne et une allégeance plus forte que le reste. Sans doute le référendum du 18 septembre 2014 sur la question de l'indépendance écossaise l'avait-il conforté dans cette voie. En pleine campagne référendaire, c'est pourtant une image radicalement différente du parti qui se dessine à lui. Au printemps 2016, le parti, paralysé par les tensions 
internes, se cristallise autour des figures médiatiques de Michael Gove et du maire de Londres, Boris Johnson, proches du Premier ministre, auxquels finiront par se joindre des figures moins connues comme Andrea Leadsom ou Priti Patel, qui incarnent l'ampleur du mouvement en faveur du retrait par rapport aux engagements européens de Cameron.

3 A l'approche du référendum, le groupe parlementaire est quant à lui scindé en deux, avec 133 députés faisant officiellement campagne pour le Brexit, soit $40 \%$ du groupe parlementaire (127 députés et 6 membres du Cabinet ${ }^{2}$ ). Cette configuration n'est que le résultat inévitable d'une évolution historique que l'on pourrait faire remonter à la ratification du traité de Maastricht lorsqu'une poignée de rebelles eurosceptiques ont fait entendre leur voix avec vigueur, menaçant la cohésion du parti et bafouant l'autorité du Premier ministre John Major qui, humilié, leur attribuera l'étiquette de "salauds" qu'ils transformeront en titre de gloire. Si certains d'entre eux sont toujours présents en 2015 sur les bancs de la Chambre des Communes, ce ne sont pas eux qui constituent le gros bataillon des partisans du retrait mais des députés plus jeunes, élus en 2010, voire en 2015 dont on aurait pu s'attendre à ce que, impressionnés par leur nouvelle fonction, ils soient plus loyaux.

4 Au moment du référendum de juin 2016, qui sont donc les partisans du Brexit au sein du groupe parlementaire conservateur? Et comment expliquer que l'euroscepticisme, que l'on a tendance à associer à l'ancienne génération des rebelles anti-Maastricht, dont le visage est essentiellement masculin, blanc et d'âge mûr, se retrouve aujourd'hui chez un nombre croissant de députés jeunes, femmes ou issues des minorités ethniques, pour la plupart élus en mai 2015 ?

5 Cet article cherchera à dévoiler le nouveau visage de l'euroscepticisme et à tenter d'expliquer la nature et les raisons de cette transformation. Si les causes exogènes liées à l'évolution du processus d'intégration européenne sur fond de crise économique sont parfois mises en avant pour expliquer la progression fulgurante de l'opposition antieuropéenne au sein du parti, elles ne nous semblent pas suffisantes pour expliquer l'ampleur du phénomène, qui ne touche plus seulement les anciens mais concerne désormais toutes les catégories socio-démographiques du groupe parlementaire, et en particulier les nouveaux élus. Ce sont, à nos yeux, les causes endogènes liées aux profondes transformations qui ont été entreprises au sein de l'organisation partisane qui expliquent le mieux à la fois les nouveaux rapports de force qui se jouent entre les différentes composantes du parti et leurs conséquences sur l'influence, le comportement et le positionnement idéologique des députés.

\section{Comment mesurer le " phénomène » eurosceptique depuis Maastricht}

6 L'histoire du parti conservateur est ponctuée par les dissensions intrapartisanes sur l'enjeu européen, au point que le terme d'eurosceptique a dès les années $1980^{3}$ été utilisé pour qualifier les opposants au projet européen. Depuis, le terme d'euroscepticisme, néologisme désormais intégré dans les dictionnaires, a fait l'objet d'une littérature scientifique foisonnante qui s'articule autour de trois axes : les partis politiques, l'opinion publique, la presse et autres organisations. Le socle commun en sont les définitions de Taggart et Szczerbiak qui font figure, dans la communauté scientifique, de référence incontournable autour de la distinction entre un euroscepticisme radical («hard») et 
modéré («soft ») :

hard Euroscepticism which might be defined as principled opposition to the project of European integration as embodied in the $E U$, in other words, based on the ceding or transfer of powers to supranational institutions such as the EU. ${ }^{4}$

A l'inverse, c'est ainsi que les auteurs définissent le "soft Euroscepticism" :

there is not a principled objection to the European integration project of transferring powers to a supranational body such as the EU, but there is opposition to the EU's current or future planned trajectory based on the future extension of competencies that the EU is planning to make"

Les auteurs, implantés à l'Université de Sussex, ont alors créé un institut de recherche, le Sussex European Institute, devenu chaire d'excellence Jean Monnet, qui fait aujourd'hui école. L'euroscepticisme partisan (Party-based Euroscepticism) est un des principaux axes de recherche de ce que Cas Mudde appelle la "Sussex School ", développant un grand nombre d'études de cas parmi lesquels les divisions du parti conservateur britannique au moment de la ratification du traité de Maastricht font figure de cas fondateur ${ }^{7}$.

7 Il y a près de vingt ans, lorsque la question n'était pas encore étudiée, nous avions proposé une définition qui présentait l'euroscepticisme conservateur «non seulement comme un discours à l'égard de l'intégration européenne mais également comme un comportement parlementaire distinct $\|^{8}$, ou encore un phénomène ${ }^{9}$. Cette double dimension avait d'ailleurs trouvé un écho rassurant dans l'étude réalisée en 2002 par Timothy Heppell ${ }^{10}$ sur l'hypothèse d'un factionnalisme conservateur engendré par Maastricht puis, plus largement, par les travaux ultérieurs de Taggart et Szczerbiak qui font la distinction entre les motivations «idéologiques-programmatiques » et «stratégiques-tactiques » de l'euroscepticisme partisan ${ }^{11}$.

8 Il est tentant de reprendre cette définition originelle pour étudier la progression de l'euroscepticisme conservateur en partant du postulat que, loin d'être une attitude complexe et difficile à isoler, il s'identifie et se mesure - avec des outils législatifs donnés comme un type de comportement parlementaire, qualifié de rébellion et identifié par le fait de voter contre la ligne du parti sur un projet de loi. Le critère de la rébellion parlementaire nous a ainsi permis de mesurer avec précision un phénomène qui à bien des égards demeurait difficile à saisir et à cerner. Spécialiste du comportement parlementaire des députés, Philip Cowley n'a eu de cesse de démontrer l'indépendance croissante des députés attestée par l'augmentation record des rébellions parlementaires dans les dernières législatures: alors qu'elles n'ont jamais dépassé les $10 \%$ dans les 4 législatures d'après guerre, avant d'augmenter dans les années 1970 puis de diminuer sous les gouvernements Thatcher et Blair, elles sont passées de $8 \%$ de 1997 à 2010, à $28 \%$ de 2005 à 2010 avant d'atteindre le chiffre record de $39 \%$ de 2010 à 2014 ${ }^{12}$, certaines ayant été d'une ampleur inégalée, en particulier celle d'octobre 2011 relative aux demandes de référendum sur le maintien dans l'Union européenne. Depuis la première candidature du pays à la CEE en 1961, les rébellions sur l'enjeu européen se sont amplifiées : en 1961, un seul député s'est rebellé contre l'adhésion et 24 se sont abstenus. En 1972, 39 députés ont voté contre l'adhésion. Dans les années 1990 marquées par la ratification du traité de Maastricht, 50 députés se sont rebellés sur 62 divisions. En octobre 2011, 81 députés ont voté une motion exigeant la tenue d'un référendum sur le maintien éventuel du Royaume-Uni dans l'Union européenne ${ }^{13}$. Constituée de plus du quart du groupe parlementaire, il s'agit de la rébellion la plus importante jamais observée sur la question européenne, et l'une des plus importantes, quel que soit l'enjeu, depuis $1945^{14}$. 
9 Mais la rébellion parlementaire n'est pas le seul mode d'expression du désaccord au sein de la Chambre des Communes: le dépôt de motions parlementaires, communément appelées Early Day Motions (EDMs) qui se présentent comme des pétitions signées par un nombre significatif de députés, en est un autre. En 1992, 84 députés conservateurs ont signé la fameuse motion du "nouveau départ" avant de créer le Fresh Start group de rebelles anti-Maastricht. Récemment, les motions ont été relativement peu nombreuses et surtout peu mobilisatrices ${ }^{15}$ car de 2010 à 2015, les rébellions parlementaires sur l'intégration européenne sont restées fréquentes, si bien que le parti a pu déployer une palette de stratégies pour gérer ces dissensions: allant des modes de sélection des candidats, contrôlés par le leadership, aux sanctions, promotions et rétributions, en passant par l'intimidation et les mesures disciplinaires pratiquées par les whips.

$10 \mathrm{Au}$ delà des stratégies parlementaires, l'expression de l'euroscepticisme a été également mesurée plus récemment par des études par questionnaires permettant d'identifier les attitudes des députés. Les travaux de Timothy Heppell ont ainsi permis d'identifier quantitativement mais aussi qualitativement le poids respectif des différentes catégories d'attitudes qualifiées d'europhiles, agnostiques et eurosceptiques. Reprenant la distinction de Taggart et Szczerbiak précédemment évoquée, Heppell identifie ainsi deux degrés de positions favorables au projet européen (Europhiles, Euroagnostics) et deux degrés distincts de positions défavorables (Soft Eurosceptics, Hard Eurosceptics). Ainsi, les eurosceptiques constituaient $58 \%$ du groupe parlementaire dans la législature 1992-97 (soit 192 députés) ${ }^{16}, 85 \%$ de 1997 à 2001 alors que le parti était dans l'opposition, soit 140 députés puis $90 \%$ du parti de 2001 à $2005^{17}$ et encore $77 \%$ en $2010^{18}$ alors que le parti est au gouvernement. Heppell démontre ainsi comment la question de l'intégration européenne qui, dans sa complexité intrinsèque, croise des questions de souveraineté et des enjeux économiques, a été le déterminant idéologique du conservatisme dans les années $1990^{19}$.

\section{La radicalisation eurosceptique comme changement de paradigme}

11 Si l'euroscepticisme est donc devenu la position officielle et majoritaire du parti, le terme même ne désigne plus la même chose: alors que le scepticisme envers l'intégration européenne à marche forcée semblait être la définition initiale du concept au milieu des années 1980, dans les années 1990 il réunissait les opposants au traité de Maastricht et à l'Union économique et monétaire et désigne aujourd'hui les députés partisans d'un retrait du Royaume-Uni de l'Union européenne. Le signifiant est resté le même mais son signifié s'est radicalisé. Le terme rassemble désormais à la fois les eurosceptiques de l'ancienne génération, comme Bill Cash ou John Redwood, qui représentaient une minorité marginalisée et ont progressé vers des prises de position plus radicales, et ceux de la nouvelle génération, ces enfants de la crise et du post-thatchérisme qui n'ont pas vécu la période où l'Europe, sans pour autant faire rêver, pouvait encore représenter une perspective de prospérité et de croissance et qui n'ont plus peur de brandir le retrait comme un nouvel espoir. Dès lors l'euroscepticisme est devenu un phénomène transgénérationnel.

12 Si le parti conservateur se révèle plus eurosceptique encore à chaque élection, la radicalisation eurosceptique se mesure à l'aune de deux indicateurs: les attitudes des 
nouveaux élus et les changements d'attitudes des anciens. Depuis la fin des années 1990, les travaux de Timothy Heppell s'appuient une enquête réalisée auprès des élus conservateurs pour connaître leurs positions sur différents enjeux relatifs à la politique économique et au rôle de l'Etat, aux questions morales et à l'intégration européenne. Comme nous l'avons déjà évoqué, il identifie aussi quatre groupes d'attitudes envers l'intégration européenne: les europhiles (classés 1), les euro-agnostiques (2), les eurosceptiques modérés ou «soft » (3) critiquant le projet européen, sans remettre en cause l'appartenance à l'Union européenne, et les eurosceptiques radicaux («hard») préconisant le retrait de l'UE. Déjà en 2012, Cowley et Stuart expliquent que les anciennes lignes de fracture entre pro-européens et eurosceptiques ne sont plus valables et que dorénavant le clivage est structuré en termes de degrés d'euroscepticisme ${ }^{20}$. Au moment de la rébellion parlementaire d'octobre 2011, montrent-ils, les pro-européens sont devenus quasi-inexistants et le clivage s'articule autour du type d'opposition au projet européen. Il reste néanmoins délicat d'identifier avec précision ce passage d'un euroscepticisme modéré vers un positionnement radical.

Une étude réalisée par Guido Fawkes, un blog conservateur indépendant ${ }^{21}$ permet d'identifier le positionnement des députés conservateurs face au Brexit. En croisant, fin mars 2016, ces données avec les travaux d'Heppell, à quelques jours du lancement officiel de la campagne référendaire, on voit se dessiner deux résultats intéressants: l'un confirme que les nouveaux élus de 2015 sont majoritairement plus eurosceptiques que leurs aînés, l'autre révèle que proportionnellement il y a beaucoup plus d'anciens élus eurosceptiques, voire opposés à l'UE, qui ont fini par se rallier à l'adhésion que de députés plutôt europhiles, voire agnostiques pour reprendre la terminologie de Heppell faisant finalement campagne pour le retrait, ce qui vient infirmer l'idée d'une radicalisation eurosceptique chez les députés élus déjà en place. Parmi ceux-ci, Heppell avait pu en effet distinguer en $201076,8 \%$ de députés eurosceptiques dont 50,3\% de modérés (3) et $26,3 \%$ de radicaux $(4)^{22}$. Ainsi sur les 256 députés déjà élus en 2015 (auxquels se sont ajoutés 74 nouveaux élus en 2015), on observe une cohérence idéologique significative, dans la mesure où 158 d'entre eux défendent aujourd'hui des positions similaires à ce qu'ils préconisaient en 2010 : les députés europhiles et euro-agnostiques militant pour le maintien dans l'UE, les députés eurosceptiques pour le retrait. Mais pour les 98 députés restant, ce n'est pas le cas. Or pour ce groupe de députés, le mouvement majoritaire est celui d'un ralliement au maintien, qui peut être analysé soit comme un assouplissement idéologique par rapport à l'enjeu européen, soit comme le signe d'une loyauté persistante envers leur leader ${ }^{23}$. Ainsi, sur les 98 députés, 85 de ceux qui avaient été classés eurosceptiques (dont 76 modérés et 9 ultra) ont défendu le maintien. Seuls 13 députés ont effectué le chemin inverse: 1 europhile (Charlotte Leslie) et 12 agnostiques ont finalement opté pour le retrait.

Ces données montrent que l'hypothèse d'une radicalisation eurosceptique des députés déjà en place en 2015 ne se vérifie donc pas dans la mesure où sur ces 256 élus lors des scrutins précédents, on trouve 100 députés qui font campagne pour le retrait (soit $39 \%$ ) et $156(61 \%)$ pour le maintien. A ces 100 Brexiteers, on peut ajouter 31 députés (41,9\%) sur les 74 élus en 2015. Proportionnellement, on dénombre donc sensiblement plus de députés qui ont fait campagne pour le retrait parmi les nouveaux élus que chez les anciens, ce qui pourrait venir confirmer l'hypothèse non pas que ces nouveaux députés sont plus rebelles (Cameron ayant permis la liberté de vote au sein du Cabinet et du 
groupe parlementaire), mais qu'ils sont bien sensiblement plus proches d'un euroscepticisme radical (« hard ») que modéré («soft »).

\section{Des députés moins rebelles et plus représentatifs}

Dès 2012, les conclusions de Cowley et Stuart semblaient pour ainsi dire visionnaires : sur les 81 rebelles d'octobre 2011, 49 (soit $59 \%$ ) étaient des députés qui venaient d'être élus en 2010, ce qui confirmait l'hypothèse selon laquelle les députés seraient de plus en plus indépendants et enclins à la rébellion et suggérait l'idée que chaque nouvelle génération était plus hostile au maintien dans l'Union européenne que la précédente. En réalité, l'idée selon laquelle les députés sont de plus en plus rebelles à chaque législature ne se vérifie pas pour la génération actuelle. Le site public whip montre des taux proches de $0 \%$ pour les nouveaux élus ${ }^{24}$. Leur euroscepticisme n'est donc pas à rechercher dans une défiance croissante envers le leader mais dans la solidité et la stabilité de leurs convictions concernant l'appartenance à l'UE. Parallèlement, parmi les plus rebelles des députés conservateurs figurent ceux qui ont mené la fronde, à l'époque de Maastricht, ou plus récemment lors du vote d'octobre 2011 exigeant un référendum sur l'UE: respectivement Bill Cash (3,7 \%) ou David Nuttall (6\%).

Les études réalisées au cours des mois qui ont précédé le référendum du 23 juin 2016 ont montré que les positions défendues dès le début de l'année 2016 sont restées relativement stables jusqu'au scrutin. L'étude menée par le blog Guido Fawkes indique qu'en mars 2016, à quelques jours du lancement officiel de la campagne prévu le 14 avril, le groupe conservateur comptait 163 députés favorables au maintien (soit $49 \%$ des 330 députés), 141 au retrait $(42,7 \%)$ et 26 indécis $(7,8 \%)$. Mais l'étude révèle alors des divisions bien plus nettes chez les 74 nouveaux élus et proportionnellement, un euroscepticisme plus marqué ainsi qu'une indécision plus forte : 32 députés favorables au retrait ( $43,2 \%$ des nouveaux élus), 32 au maintien (43,2\%) et 10 indécis (13,5\%) en mars 2016. A quelques jours du scrutin, la BBC divulguait la liste des députés conservateurs qui se sont rangés du côté du Brexit : 133 dont 127 de base et 6 membres du gouvernement ${ }^{25}$. Parmi eux, on dénombre, comme évoqué précédemment, 31 députés élus en 2015. Entre mars et juin 2016, une députée qui avait affiché son soutien au maintien s'est ralliée au retrait (Caroline Ansell) et deux autres ont effectué le chemin inverse (Seema Kennedy and Kelly Tolhurst). La corrélation de ces deux constats (des députés moins rebelles mais aussi plus eurosceptiques) offre l'image d'un euroscepticisme d'un genre nouveau. A l'inverse de leurs aînés, dont certains n'osaient pas s'avouer eurosceptiques de peur d'être assimilés au groupe de rebelles prêts à mettre en péril le gouvernement Major, cette nouvelle génération affiche un euroscepticisme décomplexé mais qui n'est pas brandi comme un instrument de contestation du gouvernement en place. De ce fait, on peut dire que leur euroscepticisme est plus idéologique que stratégique à l'inverse des rebelles de Maastricht qui ont ensuite prospéré sur le terrain de la protestation intra-partisane.

17 L'euroscepticisme conservateur à la Chambre des Communes est habituellement perçu comme le reflet de plus en plus fidèle de l'ampleur du phénomène existant à la base du parti, c'est-à-dire chez les adhérents et les militants dans les circonscriptions. C'est cette hypothèse qui a d'ailleurs guidé David Cameron lorsqu'il a lancé un avertissement en direction des députés, leur demandant expressément d'ignorer les sections locales eurosceptiques"26 provoquant la colère des grassroots et par contrecoup, des députés. Cette vision est traditionnellement répercutée par les députés eurosceptiques eux- 
mêmes, qui échangent souvent avec leurs militants les plus véhéments sur les questions européennes. L'étude menée par Tim Bale et Paul Webb sur les adhérents conservateurs offre cependant une image beaucoup plus contrastée des militants, comprenant un grand nombre d'indécis $(64,59 \%)$ qui attendaient l'issue des négociations au moment où le questionnaire leur a été envoyé et seulement $15,57 \%$ de favorables au retrait contre $19,58 \% .{ }^{27}$ Un nouveau sondage YouGov réalisé du $1^{\mathrm{er}}$ au 4 juillet 2016 auprès de 994 adhérents conservateurs a révélé que 660 d'entre eux (soit 66,4\%) avaient voté pour le retrait du pays ${ }^{28}$. Au moment de la campagne pour l'élection du leader conservateur en juillet 2016, la grande majorité des adhérents a également plébiscité Theresa May comme la candidate de la stabilité alors que celle-ci avait défendu le maintien du pays dans l'Union européenne pendant la campagne.

Le positionnement de la base électorale du parti est, quant à lui, comparable à ces données. Si l'on se fie donc aux attitudes des nouveaux élus de 2015, celles-ci seraient aussi plus conformes à celles de leurs électeurs. Un sondage YouGov indique en effet que $39 \%$ des électeurs conservateurs se sont déclarés pour le maintien et $61 \%$ pour le retrait ${ }^{29}$, à l'inverse du groupe parlementaire dans son ensemble mais plus en adéquation avec les nouveaux élus, ce qui témoigne, chez ces derniers, d'une indépendance croissante visà-vis de la ligne définie par le dirigeant, mais aussi d'une meilleure représentativité de l'électorat. Cette dépendance croissante envers l'électorat a été considérablement favorisée par un ensemble de réformes organisationnelles qui visent à resserrer le lien entre l'élu et le citoyen afin de répondre aux critiques croissantes d'un hiatus entre l'élite politique et le peuple, sur lesquelles s'appuient les arguments populistes, notamment ceux du UKIP ${ }^{30}$.

\section{Des explications structurelles}

Pour expliquer l'euroscepticisme, on évoque souvent l'évolution du projet européen face à la crise économique. Or les explications de type idéologique restent, à nos yeux, peu convaincantes pour comprendre le phénomène eurosceptique au sein du parti conservateur car elles reviendraient à considérer les députés comme des citoyens ordinaires, sans prendre en compte le poids des structures parlementaires et partisanes qui pèsent sur eux et la part stratégique des choix qu'ils sont amenés à faire dans le cadre de leur fonction. Face à une pratique gouvernementale qui a changé et se révèle plus sensible au populisme ${ }^{31}$, les députés sont aussi l'expression des transformations institutionnelles et partisanes qui affectent leurs structures. C'est la raison pour laquelle il nous semble que l'évolution des attitudes parlementaires envers la question européenne s'explique de manière beaucoup plus convaincante par l'impact de ces transformations sur le statut et la fonction parlementaire. Autrement dit, l'euroscepticisme a changé en premier lieu parce que les députés ont changé.

On peut interpréter l'euroscepticisme comme l'une des manifestations d'un discours de désaffection envers la classe politique, qui se diffuse de plus en plus et n'épargne pas les partis politiques britanniques. Il est toutefois également très présent en Europe et aux États-Unis. Le message du hiatus, omniprésent dans la campagne référendaire chez les partisans du Brexit, entre l'élite et la base, est, depuis longtemps, intégré par les partis majoritaires qui ont conduit un certain nombre de réformes pour tenter de pallier cette faille et s'inscrire, dans le même temps, dans un mouvement européen plus vaste de transformations partisanes vers toujours plus de démocratisation. En France, en Italie, en 
Grèce et au Portugal, mais aussi au Royaume-Uni, les partis politiques tentent de répondre à la nécessité d'une participation citoyenne plus active qui, à bien des égards, prend la forme d'une véritable injonction délibérative, pour reprendre l'expression de Rémi Lefebvre et Antoine Roger ${ }^{32}$, comme l'illustre par exemple le recours croissant aux primaires fermées (inclusion des adhérents et militants dans la procédure décisionnaire) ou ouvertes (inclusion des sympathisants et électeurs) pour l'élection de leur dirigeant. Le cas du parti travailliste ayant permis l'élection du dirigeant Jeremy Corbyn en septembre 2015 grâce au soutien des sympathisants qui ont pu s'enregistrer en ligne moyennant $3 £$, en est un exemple criant.

21 Mais ce mouvement a également été engagé par le parti conservateur, dont la porosité croissante avec l'électorat explique aussi comment l'euroscepticisme a progressé si vite chez des élus. Depuis les réformes engagées en 1998 par William Hague, le parti conservateur répond de diverses manières à cet impératif participatif qui traverse nos démocraties occidentales ${ }^{33}$. Trois réformes illustrent de manière significative l'évolution du parti dans ce sens et expliquent ainsi cette dépendance croissante des députés envers leurs électeurs, dans un parti dont la loyauté ne semble plus être l'arme secrète. L'introduction de primaires fermées permettant aux adhérents de départager, par scrutin postal, les deux derniers candidats en lice a permis en 2005 l'élection à la tête du parti de David Cameron pourtant peu soutenu par les députés. Si l'introduction des militants dans le processus d'élection du leader n'a pas pour autant dépossédé les députés de leurs prérogatives, ceux-ci sont en revanche devenus plus dépendants des militants pourtant de moins en moins présents sur le terrain. En mai 2005, la décision prise par Grant Shapps d'ouvrir le parti aux sympathisants (Team2015) pour pallier l'absence de militants dans certaines sections locales, illustre également l'esprit d'ouverture aux citoyens qui guide les changements organisationnels. Enfin, l'introduction de primaires ouvertes pour la sélection de candidats parlementaires dans une vingtaine de circonscriptions, bien qu'expérimentale, est un troisième exemple significatif ${ }^{34}$ de l'émergence d'un nouveau type de député, parfois émanant directement de la société civile, pas ou peu professionnalisé et plus représentatif de la base électorale. Ces transformations ont indéniablement contribué à façonner un député qui se fond mieux dans la base militante et électorale et parvient à intégrer plus facilement ses orientations idéologiques.

\section{Conclusion}

L'observation des nouveaux élus eurosceptiques nous invite à renouveler nos grilles d'analyse de l'euroscepticisme et à dépasser les motivations purement idéologiques qui font de l'évolution de l'Union européenne dans un contexte de crise la cause principale de la radicalisation eurosceptique. Si l'euroscepticisme constitue, à l'époque de Maastricht, non seulement une attitude mais un comportement parlementaire distinct, il dépasse désormais le cadre de cette analyse. L'émergence d'un nouveau type d'euroscepticisme qu'on pourrait qualifier d'euroscepticisme idéologique ou d'orthodoxie eurosceptique, caractérisant des députés qui restent toutefois fidèles au leader, introduit un nouveau clivage au sein du groupe parlementaire entre des eurosceptiques stratégiques qui trouvent dans la question européenne un motif parmi d'autres de diverger, voire de s'opposer à la position du leadership, et des eurosceptiques idéologiques qui, bien que profondément loyaux, considèrent l'enjeu européen comme suffisamment important pour faire valoir, malgré leur réticence, leur opinion divergente. 
23 Au lendemain du référendum, les divergences portent désormais non plus sur le Brexit qui fait désormais presque consensus, mais sur le type de relation que les conservateurs envisagent entre leur pays et l'Union européenne. La nouvelle ligne de clivage s'articule désormais autour du marché unique, avec l'émergence d'un nouveau groupe de députés baptisés Leave Means Leave prêts à quitter le marché unique pour combattre l'immigration, leur priorité absolue. Il reste à savoir si ces députés utiliseront stratégiquement - la rébellion parlementaire pour faire valoir leur point de vue ou s'ils chercheront dans les associations et motions un moyen plus orthodoxe d'exprimer leur scepticisme sans dévier de la ligne imposée par l'équipe dirigeante. Dans tous les cas, il est à parier que l'euroscepticisme, loin de disparaître, continuera à peser sur la vie politique britannique même lorsque le pays aura quitté l'UE.

Agrégée d'anglais et docteur en science politique de l'Institut d'Études Politiques de Paris, Agnès Alexandre-Collier est Professeur de civilisation britannique contemporaine à l'Université de Bourgogne Franche-Comté (Dijon). Spécialiste des relations entre le Royaume-Uni et l'Union européenne et de la vie politique britannique, elle est notamment l'auteur des trois ouvrages suivants : La Grande Bretagne eurosceptique? L'Europe dans le débat politique britannique (Éditions du Temps, 2002) ; Les habits neufs de David Cameron. Les conservateurs britanniques 1990-2010 (Presses de Sciences Po, 2010) et Les partis politiques en Grande-Bretagne, en collaboration avec Emmanuelle Avril (Paris, Armand Colin, 2013).

\section{BIBLIOGRAPHIE}

ALEXANDRE-COLLIER, A. « Le phénomène eurosceptique au sein du parti conservateur britannique », Politique Européenne, $\mathrm{n}^{\circ}$ 6, 2002, p. 53-73.

ALEXANDRE-COLLIER, A. « Le phénomène eurosceptique au Royaume-Uni », Outre Terre, Revue européenne de géopolitique, $\mathrm{n}^{\circ} 41$, avril 2014, p. 100-112.

ALEXANDRE-COLLIER, A. « The Open Garden of Politics? The Impact of Open Primaries for Candidate Selection in the British Conservative Party », British Journal of Politics and International Relations, volume 18, n³, August 2016, p. 706-723.

ALEXANDRE-COLLIER, A. « How Populist was David Cameron? », Juncture, volume 23, issue 2, Autumn 2016, IPPR, Wiley, p. 116-125.

BAKER D., GAMBLE A., LUDLAM S. « Whips or Scorpions? The Maastricht vote and the Conservative Party », Parliamentary Affairs, vol. 46, 1993, n 2, p. 151-166.

BAKER D., GAMBLE A., LUDLAM S. « 1846...1906...1996 ? Conservative splits and European integration ", The Political Quarterly, vol. 64, 1993, n 4, p. 420-434.

BAKER D., GAMBLE A., LUDLAM S. « The Parliamentary Siege of Maastricht 1993: Conservative Divisions and British Ratification », Parliamentary Affairs, vol. 47, n 1, 1994, p. 37-60.

BAKER D., FOUNTAIN I., GAMBLE A., LUDLAM S. « Backbench Conservative Attitudes to European Integration », Political Quarterly, vol. 66, n² 2, 1995, p. 221-233. 
COWLEY, P. \& STUART, M. « The Cambusters: The Conservative European Union Referendum Rebellion of October $2011 »$, Political Quarterly, vol. 83, n² 2, 2012, p. 402-406.

FORD, R. \& GOODWIN, M. Revolt on the Right. Explaining support for the radical right in Britain, London \& New York: Routledge, 2014.

FORSTER A. Euroscepticism in Contemporary British Politics. Opposition to Europe in the British Conservative and Labour Parties since 1945, London \& New York: Routledge, 2002.

HEPPELL, T. « The ideological composition of the parliamentary Conservative Party 1992-97 ", British Journal of Politics and International Relations, vol. 4, n 2, 2002, p. 299-324.

HEPPELL, T. \& HILL, M. « Transcending Thatcherism? Ideology and the Conservative Party leadership election of David Cameron ", Political Quarterly, vol. 80, n 3, 2009, p. 388-399.

HEPPELL, T. « Cameron and Liberal Conservatism: Attitudes within the Parliamentary Conservative Party and Conservative Ministers », The British Journal of Politics and International Relations, vol. 15, n³, 2013, p. 340-361.

LYNCH, P. \& WHITAKER, R. « Where There is Discord, Can they Bring Harmony? Managing Intraparty Dissent on European Integration in the Conservative Party », The British Journal of Politics and International Relations, vol. 15, 2013, p. 317-339.

MUDDE C. «The Comparative Study of Party-Based Euroscepticism: the Sussex vs the North Carolina School », East European Politics, vol. 28, n², 2012, p. 193-202.

SZCZERBIAK, A. \& TAGGART, P. Opposing Europe? The Comparative Party Politics of Euroscepticism, vol. 1 \& 2, Oxford: Oxford University Press, 2008.

TOURNIER-SOL, K. « Reworking the Eurosceptic and Conservative Traditions into a Populist Narrative: UKIP’s Winning Formula? », Journal of Common Market Studies, vol. 53, n¹, 2015, p. $140-156$.

\section{NOTES}

1. Lord KILMUIR, Political Adventure: The Memoirs of the Earl of Kilmuir, London: Weidenfeld \& Nicolson, 1964.

2. BBC, «EU vote: Where the Cabinet and other MPs stand », 22 juin 2016, http://www.bbc.com/ news/uk-politics-eu-referendum-35616946.

3. Sara TULLOCH, The Oxford Dictionary of New Words. A popular guide to words in the news, Oxford University Press, 1996, p. 105-107.

4. l'euroscepticisme radical (hard euroscepticism) qui pourrait être défini comme une opposition de principe au projet d'intégration européenne, tel qu'il est incarné par l'Union européenne, fondé en d'autres termes sur la délégation ou le transfert des pouvoirs à des institutions supranationales telles que l'UE». A l'inverse, c'est ainsi que les auteurs définissent «l'euroscepticisme modéré» (soft euroscepticism) : «il n’y a pas d'objection de principe au projet d'intégration européenne axé sur le transfert des pouvoirs vers une instance supranationale telle que l'UE, mais il y a une opposition à la trajectoire actuelle ou à venir de l'Union européenne, fondée sur l'extension future des compétences que l'UE envisage".

5. Aleks SZCZERBIAK \& Paul TAGGART, Opposing Europe? The Comparative Party Politics of Euroscepticism, vol. 2, Oxford : Oxford University Press, 2008, p. 247-248.

6. Cas MUDDE, «The Comparative Study of Party-Based Euroscepticism: the Sussex vs the North Carolina School », East European Politics, vol. 28, n², 2012, p. 193-202. 
7. Voir les travaux cités en bibliographie de Baker, Gamble and Seawright, Foster, Heppell, Lynch and Whitaker, Alexandre-Collier pour n'en citer que quelques-uns.

8. Agnès ALEXANDRE-COLLIER, «Le phénomène eurosceptique au sein du parti conservateur britannique », Politique Européenne, $\mathrm{n}^{\circ}$ 6, hiver 2002, p. 53-73.

9. Agnès ALEXANDRE-COLLIER, "Le phénomène eurosceptique au Royaume-Uni », Outre Terre, Revue européenne de géopolitique, $\mathrm{n}^{\circ} 41$, avril 2014, p. 100-112.

10. Timothy HEPPELL, «The ideological composition of the parliamentary Conservative Party 1992-97 », British Journal of Politics and International Relations, vol. 4, n², 2002, p. 299-324.

11. Aleks SZCZERBIAK \& Paul TAGGART, op. cit. p. 247-248.

12. Meg RUSSELL and Philip COWLEY, «The Policy Power of the Westminster Parliament: the "Parliamentary State" and the Empirical Evidence », Governance, vol. 29, n 1, 2016, p. 125.

13. Philip LYNCH \& Richard WHITAKER, « Where There is Discord, Can They Bring Harmony? Managing Intra-Party Dissent on European Integration in the Conservative Party ", British Journal of Politics and International Relations, vol. 15, 2013, p. 318.

14. Philip COWLEY et Mark STUART, "The Cambusters: The Conservative European Union Referendum Rebellion of October 2011 », Political Quarterly, vol. 83, n² 2, avril-juin 2012, p. 402.

15. Les principales motions déposées sur les questions européennes n'ont jamais réuni pendant cette période plus d'une poignée de signataires. Voir: http://www.parliament.uk/business/ publications/business-papers/commons/early-day-motions/ Site consulté le 20 octobre 2016.

16. Timothy HEPPELL, op. cit., 2002, p. 299-324.

17. Timothy HEPPELL and Michael HILL, "Transcending Thatcherism? Ideology and the Conservative Party leadership election of David Cameron », Political Quarterly, vol. 80, n 3, 2009, p. 88-399.

18. Timothy HEPPELL, « Cameron and Liberal Conservatism: Attitudes within the Parliamentary Conservative Party and Conservative Ministers", British Journal of Politics and International Relations, vol. 15, $\mathrm{n}^{\circ} 3,2013$, p. 340-361.

19. Timothy HEPPELL, op. cit., British Journal of Politics and International Relations, vol. $4, n^{\circ} 2,2002$, p. 320.

20. Philip COWLEY et Mark STUART, op. cit, Political Quarterly, p. 402.

21. http://order-order.com/2016/03/03/141-tory-mps-backing-brexit/ Site consulté le 20 octobre 2016.

22. Timothy HEPPELL, op. cit., British Journal of Politics and International Relations, vol. $15, \mathrm{n}^{\circ} 3$, 2013, p. 340-361.

23. D'après les députés James Cleverly et Anne Marie Trevelyan, élus en 2015, cette loyauté s'explique aussi par la perspective d'une promotion plus envisageable pour les anciens que pour les nouveaux élus. Interviews à Londres les 7 et 8 septembre 2016.

24. Entre $0 \%$ et $1 \%$ (Craig Tracey) sur www.publicwhip.org (site consulté le 10 octobre 2016).

25. BBC, «EU vote: Where the Cabinet and other MPs stand », 22 juin 2016, http://www.bbc.com/ news/uk-politics-eu-referendum-35616946 (site consulté le 20 octobre 2016).

26. Norman TEBBIT, «Tory leadership hopefuls should ignore the PM and reach out to the grassroots ", The Telegraph, 4 février 2016.

27. https://proftimbale.com/2016/02/21/cameron-and-tebbit-are-both-wrong-tory-activistsare-not-as-set-on-leaving-the-eu-as-many-imagine-with-monica-poletti-and-paul-webb-5february-2016/ (site consulté le 20 octobre 2016).

28. https://d25d2506sfb94s.cloudfront.net/cumulus_uploads/document/dgak27s1eh/ TimesResults_160704_ConservativeMembers.pdf (site consulté le 20 octobre 2016).

29. https://yougov.co.uk/news/2016/06/27/how-britain-voted/ (site consulté le 20 octobre 2016).

30. Robert FORD, Mathew GOODWIN, Revolt on the Right. Explaining support for the radical right in Britain, London \& New York: Routledge, 2014. Karine TOURNIER-SOL, «Reworking the 
Eurosceptic and Conservative Traditions into a Populist Narrative: UKIP's Winning Formula?», Journal of Common Market Studies, vol. 53, n¹1, 2015, p. 140-156.

31. Agnès ALEXANDRE-COLLIER, «How Populist was David Cameron? », Juncture, volume 23, issue 2, Autumn 2016, IPPR, Wiley, p. 116-125.

32. Rémi LEFEBVRE et Antoine ROGER, (dir.) Les partis politiques à l'épreuve des procédures délibératives, Rennes: Presses Universitaires de Rennes, 2009.

33. Agnès ALEXANDRE-COLLIER, «Les conservateurs britanniques confrontés à une dérive populiste ", in : Dominique ANDOLFATTO et Alexandra GOUJON (dir.), Les partis politiques, ateliers de la démocratie, Bruxelles, Editions de l'Université Libre de Bruxelles, 2016, p. 31-42.

34. Agnès ALEXANDRE-COLLIER, « The Open Garden of Politics? The Impact of Open Primaries for Candidate Selection in the British Conservative Party ", British Journal of Politics and International Relations, volume 18, n³, August 2016, p. 706-723.

\section{RÉSUMÉS}

Cet article s'intéresse aux députés conservateurs qui ont fait campagne pour le Brexit au moment du référendum du 23 juin 2016. Après un bref survol de l'évolution du terme "euroscepticisme" et des attitudes conservatrices envers l'appartenance à l'Union européenne, l'article s'efforcera de dessiner le nouveau visage de l'euroscepticisme conservateur au sein de la chambre des Communes, en s'attardant plus particulièrement sur les nouveaux élus de 2015 qui sont censés inclure plus de femmes, de minorités ethniques et de jeunes. Grâce à une série d'indicateurs liés au comportement parlementaire, cet article montrera que les nouveaux Brexiteers sont désormais moins dépendants de l'équipe dirigeante mais aussi plus fidèles à leurs électeurs et représentatifs de leurs attitudes. Il s'agira enfin de proposer des explications possibles de ce nouveau phénomène, en s'appuyant sur les changements organisationnels au sein du parti qui ont, selon nous, ouvert la voie à l'émergence d'un nouveau type de député. En termes plus simples, si l'euroscepticisme au sein du parti a changé, c'est essentiellement parce que les députés conservateurs ont changé de manière radicale.

This article will look at the Conservative MPs who campaigned for Brexit in the run-up to the referendum on 23rd June 2016. After a brief overview of the evolution of the label Euroscepticism and attitudes towards EU membership in the party, it will first try and sketch out the new face of Conservative Euroscepticism in the House of Commons, with a special focus on the 2015 intake of MPs who are supposedly "less pale, male and stale". Through a series of indicators related to parliamentary behaviour, this article will argue that the new Brexiteers are now less dependent on party leadership but more loyal to and representative of their voters. It will finally offer tentative explanations of this new phenomenon, based on party organisational changes which arguably paved the way for the emergence of a new type of Conservative MP. To put it simply, if Euroscepticism has changed within the party, it is essentially because Conservative MPs have radically changed. 
INDEX

Mots-clés : Parti conservateur, euroscepticisme, parlement

Keywords : Conservative Party, Euroscepticism, Parliament

\section{AUTEUR}

\section{AGNÈS ALEXANDRE-COLLIER}

TIL, EA 4182, Université de Bourgogne Franche-Comté. 